\title{
EDITORIAL
}

\section{FRAILTY - AN EMERGING HIGH IMPACT COMPLICATION OF DIABETES REQUIRING SPECIFIC MANAGEMENT APPROACHES}

\author{
A. SINCLAIR ${ }^{1}$, B. VELLAS ${ }^{2}$

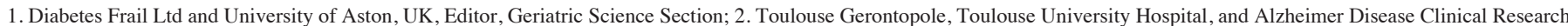 \\ Centre, University of Toulouse, France, Editor-in-Chief, JNHA. Corresponding author: Professor Alan Sinclair, Foundation for Diabetes Research in Older People, Diabetes Frail Ltd, \\ Medici Medical Practice, Luton LU1 3UA, UK; Tel: 0044 (0) 1582 738464, Email: ajsinclair@ diabetesfrail.org
}

\section{Introduction}

Ever since the Frailty model for diabetes was first described (1) a different management approach to older adults with diabetes who develop frailty has been suggested. The European Diabetes Working Party for Older People (EDWPOP) identified frailty as a key priority area for action (2) and published a glucose-lowering algorithm for frail patients with type 2 diabetes. More recently, the international Diabetes Federation (IDF) launched global guidance on the management of diabetes in older adults and made specific recommendations on treatment in those adults who are dependent and frail who depend on others for their diabetes care (3). Other organisations recognise the impact of multimorbidity and frailty in the overall management of older citizens with diabetes (4-6). We are pleased to announce that more considered guidance to this complex area of diabetes management is in development and in mid-2017, an international collaboration consisting of EDWPOP, IAGG-Garn, AMDA, ICFRSR, and an International Group of Experts, will publish a Position Statement in this area* (see list of collaborators at end of article).

In planning the Position statement, the international Writing Group have acknowledged that frailty is a common finding and may be present in $32-48 \%$ of adults aged 65 years and over with diabetes (7) and is associated with adverse outcomes and reduced survival (8). At the same time, the Group have recognised the scarcity of specific studies of glucose-lowering treatments in older patients with frailty and diabetes (9), the lack of an operational definition of frailty, the urgent need for health professionals involved in diabetes care to acquire new skills and competencies in assessment of functional status, and the need for education and practical guidance for clinicians in managing frailty in those with diabetes. The Position Statement will point out the limitations of pharma-directed, short duration studies (10) and emphasise the need for a wider application of the concept of frailty in the clinical arena, a greater use of frailty assessments in clinical diabetes practice (for example, recommending simple, pragmatic tools to identify patients at risk, the development of specific clinical trial methodology for frailty in diabetes, and a renewed emphasis on how we influence commissioners (health boards, primary care organisations) of clinical services to purchase care pathways for frailty in their diabetic populations (11).

Recent studies that have attempted to study the benefits of treating glycaemia with DPP4-inhibitors in older adults with multimorbidity or frailty $(9,10)$ point out the advantages in treatment such as a reduced risk of hypoglycaemia and no appreciable weight gain. These are indeed important aspects of individualised care in this class of glucose-lowering therapy but future studies involving a wider spectrum of agents need to discuss precisely the description of $\mathrm{HbAlc}$ targets, and how these should be managed, achieved, and what clinical relevance they have. The use of fasting glucose levels to guide treatment decisions is also questionable in older patients. Attempts to lower HbA1c towards $7 \cdot 0 \%$ (53 mmol/mol) or less in an older cohort of patients with diabetes might seem unnecessary or even dangerous since higher target levels are now recommended $(2,4,6)$ and more liberal targets in those who are frail are now recommended (3). Such practical issues in management are important to a clinician and those in primary care and community care settings need to be convinced of their importance, otherwise they will not be acted upon.

Relevant outcome measures will also prove to have an important influence in deciding if a specific management strategy is worthwhile in routine clinical care. Apart from glycaemia targets, two other key outcomes that require assessment but usually are not a feature of every day clinical practice are quality of life and functional health status indicators but to introduce these measures will require a culture change by the diabetes healthcare team and a phase of upskilling in assessment procedures. Other outcomes such as rates of admission to hospital, falls rate, changes in cognition and balance, and other functional measures such as walking speed need to become a routine part of the annual review process.

Sarcopenia is associated with diabetes (7) and may be an integral phase towards the development of frailty (12). Muscle loss is also accelerated in diabetes (13) and lower limb muscles may be in a profound state of insulin resistance (14). As a consequence, the clinician must now also consider the use of insulin sensitisers combined with weight loss and resistance exercise to improve muscle function in frailty (7). Clinical trial evidence of the benefits or not of resistance training in older people with diabetes and frailty will be available in Mid-2017 


\section{AN EMERGING HIGH IMPACT COMPLICATION OF DIABETES REQUIRING SPECIFIC MANAGEMENT APPROACHES}

when the results of the European Union-funded MIDFRAIL study ae released (15).

What has not been worked out yet is who will take the lead locally in a community in bringing about these management approaches although it is hoped that both hospital specialists and primary care physicians will take a joint action in implementation. We hope that the work of our International Writing Group will at least provide a starting point to offer guidance to optimise the clinical care of all older adults with diabetes and frailty.

* Collaborators: European Diabetes Working Party for Older People (EDWPOP), International Association of Gerontology and Geriatrics (IAGG)-GARN (Global Aging Research Network); American Medical Directors Association (AMDA), and the International Conference on Frailty \& Sarcopenia Research (ICFSR), and an International Group of Experts

Conflict of Interest: None

\section{References}

1. Sinclair AJ. Diabetes in old age-changing concepts in the secondary care arena. J R Coll Physicians Lond 2000; 34: 240-44.

2. Sinclair AJ, Paolisso G, Castro M, Bourdel-Marchasson I, Gadsby R, Rodriguez Mañas L, European Diabetes Working Party for Older People. European Diabetes Working Party for Older People 2011 clinical guidelines References for type 2 diabetes mellitus. Executive summary. Diabetes Metab 2011;37 (suppl 3): S27-38.

3. Dunning T, Sinclair AJ, Colagiuri S. New IDF Guideline for managing type 2 diabetes in older people. Diabetes Res Clin Pract. 2014 Mar;103(3):538-40.

4. Inzucchi SE, Bergenstal RM, Buse JB, et al. Management of hyperglycemia in type 2 diabetes: a patient-centered approach: position statement of the American Diabetes Association (ADA) and the European Association for the Study of Diabetes (EASD). Diabetes Care 2012; 35: 1364-79.
5. Kirkman MS, Briscoe VJ, Clark N, et al. Diabetes in older adults.Diabetes Care 2012; 35: 2650-64.

6. Sinclair A, Morley JE, Rodriquez-Manas L, et al. Diabetes mellitus in older people: position statement on behalf of International Association of Gerontology and Geriatrics (IAGG), the European Diabetes Working Party for Older People (EDWPOP), and the International Task Force of Experts of Diabetes. J Am Med Dir Assoc 2012; 13: 497-502.

7. Morley JE, Malmstrom TK, Rodriguez-Mañas L, Sinclair AJ. Frailty, sarcopenia and diabetes. J Am Med Dir Assoc. 2014 Dec;15(12):853-9

8. Castro-Rodríguez M, Carnicero JA, Garcia-Garcia FJ, Walter S, Morley JE, Rodríguez-Artalejo F, Sinclair AJ, Rodríguez-Mañas L. Frailty as a Major Factor in the Increased Risk of Death and Disability in Older People With Diabetes. J Am Med Dir Assoc. 2016 Oct 1;17(10):949-55.

9. Barnett AH, Huisman H, Jones R, von Eynatten M, Patel S, Woerle H-J. Linagliptin for patients aged 70 years or older with type 2 diabetes inadequately controlled with common antidiabetes treatments: a randomised, double-blind, placebo-controlled trial. Lancet 2013; published online Aug 13. http://dx.doi.org/10.1016/S01406736(13)61500-7.

10. Strain WD, Lukashevich V, Kothny W, Hoellinger MJ, Paldánius PM. Individualised treatment targets for elderly patients with type 2 diabetes using vildagliptin add-on or lone therapy (INTERVAL): a 24 week, randomised, double-blind, placebo-controlled study. Lancet 2013; 382: 409-16.

11. Sinclair A, Morley J. Frailty and diabetes.. Lancet. 2013 Oct 26;382(9902):1386-7. doi: 10.1016/S0140-6736(13)61676-1. Epub 2013 Aug 13.

12. Sinclair AJ, Rodriguez-Mañas L; Diabetes and Frailty: Two Converging Conditions? Can J Diabetes. 2016 Feb;40(1):77-83

13. Park SW1, Goodpaster BH, Strotmeyer ES, Kuller LH, Broudeau R, Kammerer C, de Rekeneire N, Harris TB, Schwartz AV, Tylavsky FA, Cho YW, Newman AB; Health, Aging, and Body Composition Study. Accelerated loss of skeletal muscle strength in older adults with type 2 diabetes: the health, aging, and body composition study. Diabetes Care. 2007 Jun;30(6):1507-12. Epub 2007 Mar 15.

14. DeFronzo RA. Skeletal Muscle Insulin Resistance Is the Primary Defect in Type 2 Diabetes. Diabetes Care 2009 Nov; 32(suppl 2): S157-S163

15. Rodríguez-Mañas L, Bayer AJ, Kelly M, Zeyfang A, Izquierdo M, Laosa O, Hardman TC, Sinclair AJ; MID-Frail Consortium. An evaluation of the effectiveness of a multi-modal intervention in frail and pre-frail older people with type 2 diabetes--the MID-Frail study: study protocol for a randomised controlled trial. Trials. 2014 Jan $24 ; 15: 34$ 